\title{
ETUDE DE LA STRUCTURE DES NOYAUX PAR REACTIONS DE TRANSFERT : IONS LEGERS ET IONS LOURDS
}

\author{
soussel, P.
}

Institut de Physique Nucléaire, 3.P. N¹, 91406 Orsay, Cedex.

Résumé : On examine quels sont les f́iéments de la structure nucléaire accessıbles par réactıon de transfert. On insiste sur le lifn entre la sélectivité de la réaction, son mécanisme et la structure atteinte. Les différences entre projectiles lourds et légers sont examinées en développant particulièrement l'analyse semi-classique du transfert qui met en évidence l'importance de la dynamique de la réaction et celle de l'adaptation de moment angulaire. Il apparait que les transferts à énergie par nucléon élevée (E/A > $5 \mathrm{MeV}$ au-dessus de la barrière) ont été peu explorés en ions lourds alors que la présence, dans ce cas, d'un moment orbital interne devrait leur donner une spécificité dans les structures atteintes.

Quelques exemples récents d'utilisation des réactıons de transfert sont ensuite passés en revue. On conclut en décrivant un progrès expérimental devant permettre l'amélioration de la résolution en énergle dans les transferts en ions lourds.

\begin{abstract}
The different information on nuclear structure wich can be obtained by transfer reactions are investigated. Emphasis is put on the strong bonds which connect the selectivity of a reaction, its mechanism and the populated structure. The difference between light and heavy projectiles is studied using a semi-classical analysis. It is noted that heavy-ion induced transfer well above the coulomb barrier $(E / A \geq 5 \mathrm{MeV}$ ) have not been extensively explored though they should exhibit special features due to the internal orbital angular momentum. Several recent experiments are given to examplifie the use of transfer reactions for nuclear structure studies using both light and heavy projectiles. One experimental progress in using heavy ion beams for high resolution experiments is described.
\end{abstract}

\section{I - Introduction - Généralités -}

L'étude de la structure des noyaux est cette partie de la physique nucléaire qui se restreint aux états stationnaires ou (le plus souvent) quasi-stationnaire de la matière nucléaire, par opposition à l'étude des processus où l'on étudie l'évolution dans le temps d'un système nucléaire. Parmi les états stationnaires, les noyaux sont localisés dans une région restreinte de l'espace, par opposition aux états (quasi) stationnaires de diffusion ou de bombardement qui sont associés à des ondes de dimensions indéfinies. Les réactions de transfert sont justement ceux de ces états de diffusion pour lesquels il y a réarrangement, c'est-à-dire répartition différente des nucléons à l'entrée et à la sortie ; on se limitera ici aux réactions à deux corps ou séquentielles dont la première étape est à deux corps du type :

$$
\mathrm{A}(\mathrm{a}, \mathrm{b}) \stackrel{\mathrm{B}^{*}}{\rightarrow} \mathrm{x}+\ldots
$$

on trouvera en référence (1) (2) (3) trois ouvrages très différents qui couvrent le domaine étudié.

Que cherche-t-on à apprendre sur l'état nucléaire étudié ?

D'abord un certain nombre de grandeurs correspondant à des variables quantiques : énergie (masse ou énergie d'excitation) spin $j$, parité $\pi$, spin isotopique $\mathrm{t}$ etc... Ensuite, on cherchera à connastre la fonction d'onde de cet état ou sa configuration, c'est-à-dire à mesurer la projection de l'état étudié sur les différents vecteurs de la base choisie. C'est là qu'apparaît la spécificité des réactions de transfert (qu'on illustrera pour simplicité sur le cas du stripping d'un nucléon) : mesurer la projection de l'état final $B^{*}$ sur un état obtenu en couplant un ou des états $k$ de la cible-coeur $A$ avec une ou des fonctions d'onde $\varphi_{\mathrm{nl}} \mathrm{j}$ d'un nucléon autour de ce coeur. On définit ainsi le facteur spectroscopique :

$$
S=\left\langle B^{*} \mid A+1\right\rangle \text { avec }|A+1\rangle=A \otimes \varphi_{n l j}
$$

ou plus généralement $|A+1\rangle=\sum_{k n l j} A k \otimes \varphi_{n l j}(2)$
L'expression (1) correspond au mécanisme le plus simple (ondes planes ou ondes distordues). Li-expression (2) peut en principe s'applıquer au mécanisme le plus compliqué en augmentant le nombre de termes $\mathrm{k}$ et $\mathrm{n}, \mathrm{l}, \mathrm{j}$. En fait, la réaction ne sera intêressante que si un ou seulement quelques termes contribuent c'est-à-dire si le mécanisme de la réaction est sélectif. L'expérience a montré qu'il en est s.ouvent ainsi au molns pour les sections efficaces importantes. Indiquons de plus que telle réaction de transfert peut être sélective d'une autre façon : en favorisant telle ou telle valeur de $(n), l$ ou $\dot{j}$. ou en leur donnant, par exemple, une distribution angulaire caractéristique. C'est l'une ou/ et l'autre de ces sélectivités d'unt réaction de transfert choisie qui permet de séparer et d'étudier des niveaux de configuration déterminée jusque dans des régions à très forte densité de niveaux. Par contre, dans les mesures de masse par des réactions à deux corps à très faibles sections efficaces, la sélectivité peut être une source de confusion. Ainsi, si la masse du fondamental et le début du spectre sont très mal connus ou mal prédits, on risque d'identifier le premier état observé comme étant le fondamental si celui-ci, défavorisé, n'est pas du tout peuplé. On doit insister donc, sur la nécessité de considérer ensemble les trois termes ; structure étudiée, mécanisme de la réaction utilisée et sélectivité observée.

\section{Que mesure-t-on dans une expérience de transfert ?}

Par ordre de difficulté croissante et parallèlement de richesse d'information, on peut mesurer : un spectre en énergie de la particule $b \quad \sigma\left(E_{b}\right)$, une distribution angulaire $\sigma\left(\theta_{b}\right)$, ou une fonction d'excitation $\sigma\left(E_{j}\right)$, puis une corrélation angulaire de $b$ avec un produit de désexcitation du noyau formé $B$ (1'utilisation de faisceaux polarisés ou la mesure directe de la polarisation peut conduire au meme type d'information). A chacune de ces mesures correspond un dispositif expérimental avec des qualités propres, en constant progrès pour obtenir plus d'information et sur un plus grand nombre d'états. 
Interprétation. Elle nécessite évidemment i'utilisation d'un modèle de mécanisme, sımple ou élaboré et d'autant plus élaboré que la réaction est moins sélective pour l'état considéré et pour la propriété étudiée. Le plus souvent, les conditions de l'expérience permettent de se placer dans le cadre des réactions directes qui correspondent à des processus rapides et pour lesquelles peu de degrés de liberté entrent en jeu (une variation lente de la fonction d'excitation constitue un test de ces hypothèses). Le modèle de base est l'approximation de Born en ondes déformées (D.W.B.A.) qui traite le transfert comme une perturbation au premier ordre entre les deux voies élastiques (en entrée et sortie) décrites par le modèle optique. Mais différentes modifications ont du être apportées pour tenir compte des processus d'ordre supérieur : ondes couplées

(CCBA) qui tient compte des excitations inélastiques dans les voies d'entrée et de sortie (pointillé sur la figure), transfert séquentiel (trait mixte) avec passage par un noyau intermédiaire I, "polarisation" défor-

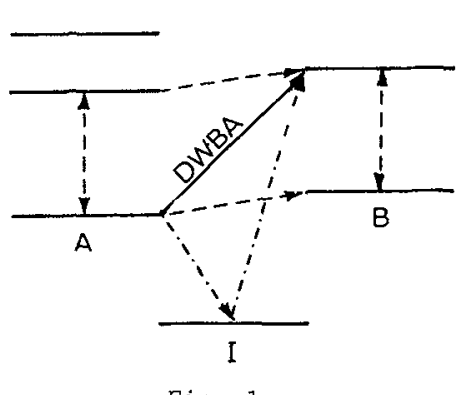

Fig. 1

mation des orbites des nucléons transférés par la proximité d'un ion lourd, enfin possibilité d'intervention de résonnances dans les voies d'entrée ou de sortie.

\section{II - Différences et similitudes entre les réactions avec projectiles légers (i.l) et projectiles lourds (I.L).}

Il n'apparaît pas que les réactions $i .1$. procèdent systématiquement par un mécanisme plus simple : en effet, si d'un côté, le nombre de degrés de liberté est à priori plus petit, par contre, les projectiles légers (aux énergies couramment pratiquées) sont plus pénétrants que les $\mathrm{I} . \mathrm{L}$. et font donc intervenir des régions profondes du noyau, moins bien connues (4) que leur surface, lieu spécifique des réactions par I.L. Les différentes sophistications du modèle de mécanisme ne sont donc pas spécifiques aux I.L., elles se développent en fait aujourd'hui de façon concertée dans les deux domaines i.l. et I.L. Il faut d'autre part remarquer que l'énorme quantité de résultats obtenus en i.l. a conduit à utiliser des "recettes" dans les analyses. Ces recettes sont partiellement fondées sur une connaissance plus étendue et plus appronfondie du domaine mais elles sont aussi fondées sur un consensus (choix de paramètres de potentiel optique par exemple) qui peut masquer parfois de réelles ambiguités. Un point cependant semble indiquer un retard théorique ou une difficulté spécifique pour les I.L. : la valeur et la dispersion du coefficient de normalisation $N$ entre les sections efficaces calculées et expérimentales. N varie de quelques unités à mille ! Terminons cet examen par deux points plus nets : - un avantage, trivial mais important, aux transferts I.L. : c'est que de nombreux multitransferts ne ${ }^{\prime}$ sont accessibles que par I.L. Pensons par exemple au stripping de quatre neutrons $\left(18_{0}, 140\right)$ ou $\left(14_{C}, 10 \mathrm{C}\right)$. Au contraire, on trouve plus souvent des distributions angulaires de forme caractéristique (du moment transféré ou du spin final) avec les réactions i.l. qu'avec celles en I.L. et la résolution en énergie reste bien meilleure en i.l. qu'en I.L.
Mais la comparaison I.L./i.l. la plus intéressante est celle des dynamiques des réactions, qu'on va examiner maintenant d'un point de vue semiclassique et en se restreignant le plus souvent. pour simplicité, au cas du stripping d'un nucléon.

\section{Transfert d'énergie de moment angulaire.}

on reprend la réaction $\mathrm{A}(\mathrm{a}, \mathrm{b}) \mathrm{B}$ avec des noyaux de masse respective $m_{2} m_{1} m_{3} m_{4}$ (transfert $n=$ $m_{1}-m_{3}$ ) et de rayons $R_{2} R_{1} R_{3} R_{4}$. On suppose le transfert entre deux trajectoires classiques

(fig. 2), on suppose aussi que tous les fragments conservent leur vitesse au cours du transfert (dans le plan de réaction) et on néglige l'énergie de liarson du cluster transféré.

Soit $\vec{l}_{i}$ le moment angulair orbital interne de $n$ dans $a$ solt $i$ la projection de $\vec{l}_{i}$ sur l'axe perpendiculaire au plan de réaction. Soit Vi le module de la vitesse relative dans la voie d'entrée : $V_{1}^{2}=2\left(E_{i}-B_{i}\right) / \mu_{i}\left(B_{i}\right.$ bar rière coulombienne, E1 énergie, $\mu_{i}$ masse rédurte, dans la voie d'entrée). On peut montrer (5) que la réaction

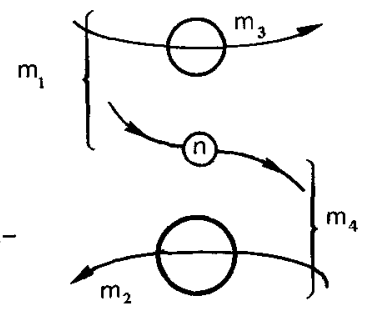
va se raire avec un transfert d'énergie et un transfert de moment angulaire dont les valeurs favorisées $Q_{f}$ et $l_{f}$ sont données par :

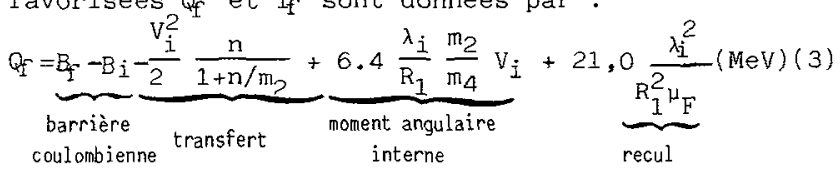

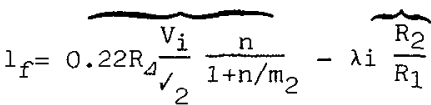

Limitons nous maintenant, pour mieux voir "fonctionner" les formules, au cas du stripping d'un neutron sur une cible lourde $\left(R_{2}=R_{4}=R\right)$ à partir, soit d'un projectile léger dans un état $s\left(l_{i}=\lambda_{i}=0\right)$, soit d'un projectile lourd $\left(1<<m_{3}<<m_{2}\right)$ de rayon $r$, dans un état quelconque. On a alors avec $\underline{e}=$ $\frac{E i-B i}{m_{1}}=\frac{V P}{2} \frac{l^{\prime} \text { énergie par nucléon au-dessus de la }}{\text { barrière. }}$

$\left.\begin{array}{l}-Q_{F} \simeq e-9.1 \lambda i \vee e / r \\ -1_{f} / R \simeq 0.22 \sqrt{e}-\lambda i / r\end{array}\right\} \stackrel{\lambda=0}{\longrightarrow}\left\{\begin{array}{l}-Q_{F}=e \\ 1_{F / R}=0.22 \sqrt{e}\end{array}\right.$

Pour le cas $\lambda=0$ on arrive ainsi à une expression simple de $Q_{f}$ et $1_{f / R}$ en fonction du seul paramètree, indépendamment donc de la nature de l'ion. On a représenté sur la figure 3 la courbe $l_{F / R}$ en fonction de $e=-Q_{f}$. On a aussi indiqué sur deux axes parallèles à e les énergies C.M. correspondantes à un cas i.1. $\left(d+64_{\mathrm{Ni}}\right.$, et un cas I.L. $\left(16_{0+} 64_{\mathrm{Ni}}\right)$; les couches atteintes dans $1 e^{65} \mathrm{Ni}$ sont indiquées sur un axe parallèle à $l_{f} / R$. On constate alors, pour les énergies le plus souvent pratiquées pour la spectroscopie, que e $<4 \mathrm{MeV} /$ nucléon en I.L. alors que e $\gtrsim 10 \mathrm{MeV} / \mathrm{nucl}^{2}$. en $i .1$. et en conséquence que $\underline{\underline{V}}_{f}$, de même que $-Q_{f}$ donc, est en fait plus élevé avec les i.l. qu'avec les I.L. !

(cela peut contredire ce qui est quelquefois cru; on verra ci-dessous un autre aspect de la comparaison i.1./I.L.) : 


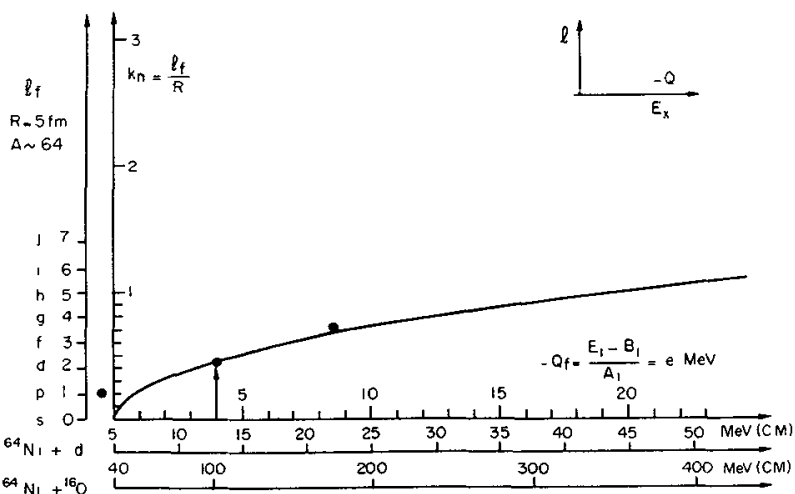

Fig. 3: Stripping d'un neutron : Variation du moment anqulaire transféré en fonction de l'énergie cinétique par nucléon au-dessus de la barrière $e=-Q f$ (voir texte).

On arriverait à des valeurs égales pour les mêmes valeurs de e (1.1. et I.L.) mais pour l'essentiel; les expériences I.L. restent à faire (I.L. à e n 5 à $15 \mathrm{MeV} / \mathrm{A}$ ).

Il est intéressant de comparer les valeurs du moment angulaire transféré $1_{\mathrm{f}}$ déduites précédemment au transfert $\Delta \mathrm{L}(Q)=\mathrm{L}_{i}-\mathrm{L}_{\mathrm{f}}(Q)$ calculé plus couramment, pour chaque $Q$, comme la différence des moments angulaires orbitaux entre la voie d'entrée et la voie de sortie. On peut montrer que :

$$
\text { pour } Q=Q_{f}\left\{\begin{array}{l}
I_{f}=\Delta L=\Delta L\left(Q_{f}\right) \\
\frac{d \Delta L}{d Q}=\frac{d l_{f}}{d Q} x / m_{3}=-\frac{d I_{f}}{d e} / m_{3}
\end{array}\right.
$$

Il apparait donc une différence entre i.l. et I.L. ( 1 e terme $\checkmark m_{3}$ ) : pour un même $Q_{f}$, un même e donc, $d \Delta L$ est plus grand en I.L. qu'en 1.1. et donc en s'éfoignant de la fenêtre en $Q$ la réaction $I . L$. permettra d'atteindre des spins plus élevés. Cet argument est renforcé sı on tient compte, qu'en fait en I.L., e est généralement plus petit et donc $\frac{d l f}{d e}$ plus grand.

\section{Influence du moment orbital interne.}

La présence d'un moment orbital interne $l_{j}$ non nul est spécifique aux lons lourds, elle se traduit par les termes en $\lambda_{i}$ dans les expressions (5) et (6) de $Q_{f}$ et $l_{f} / R$. Pour en figurer les effets, on a indiqué par une croix sur la figure 3 , pour la valeur $e=4$ et pour la valeur $r$, celle de $1.16_{0}$ les trois valeurs de 1 et $Q$ correspondant à $\lambda_{j}=-1$, $\lambda_{j}=0$ (sur la courbe donc) et $\lambda_{j}=+1$. Ce qui apparait, c'est un élargissement de la fenêtre en $Q$ (équivalent à l'utilisation d'une gamme étendue d'énergies incidentes avec $\lambda=0$ ) mais qui conserve (approximativement) la relation $l_{f}(Q)$ donnée par la courbe $l_{f}(e)$ pour $\lambda=0$. On peut le montrer directement à partir des formules (5) et (6).

\section{Cas particulier du transfert de deux nucléons.}

La spécificité du transfert de deux nucléons provient du fait qu'on peut traiter les deux nucléons de deux facons différentes : on peut les considérer comme un agregat de masse 2 , on en déduit alors une valeur de $Q_{f}$ et $l_{f}$ mais on peut aussi considérer séparément le transfert de chacun des nucléons en tenant compte du couplage de leur moment angulaire dans le projectile. Pour un i.1.. en général $1_{i}=0$ (c'est le cas pour $t, p$ par exemple) et les deux points de vue aboutissent au même résultat pour $Q_{f}$ et $l_{f}$. Avec un I.L. au contraire (pour le cas ou les deux nucléons sont dans des couches de moment orbital non nul), on est conduit à calculer $l_{f}$, avec deux valeurs de $\lambda_{1}$ donnant aussi deux valeurs différentes pour $l_{f}$ et des couches différentes pour chacun des nucléons transférés. Si les deux nucléons sont couplés à zéro on aura $\lambda_{i}=-\lambda^{\prime}{ }_{i}$ et l'écart entre les deux valeurs de $l_{f}$ et $l^{1}$ 'f sera maximum. On a illustré sur la fig. 4 l'origine physique simple de cette différence : la composition de la vitesse du projectile $V_{p}$ avec la vitesse de rotation $+V_{R}$ ou $-V_{R}$ selon que $\lambda_{l}$ à la valeur positive ou négative.

Les prédictions de calculs semi-classiques qu'on a examiné dans ce chapitre sont forcément qualitatives, on peut dire cependant qu'elles expliquent bien le comportement général des résultats expérimentaux.
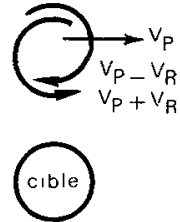

Fig. 4

\section{IV - Réactions de transfert i.l. et I.L. : quelques résultats récents.}

On va passer en revue quelques expériences qui illustrent I'utilisation spectroscopique d'une réaction de transfert. Faute de place, on indiquera seulement le but de chacune de ces expériences et les physiciens ou/et la référence à consulter. (i) comparaison des réactions $(p, t)$ et $(t, p)$ sur une série d'isotopes (6) ; cette expérience illustre la spécificité de la réaction de transfert pour mesurer un "recouvrement". Ici on peut attribuer aux niveaux peuplés l'appartenance à I'une ou l'autre de deux familles d'états distincts par la forme (sphériques et déformés par exemple). Dans le cas des germaniums, ces expériences ont permis de mettre en évidence une transition de forme entre ${ }^{72} \mathrm{Ge}$ et ${ }^{74} \mathrm{Ge}$ avec, dans chacun de ces noyaux, un $\mathrm{O}^{+}$excité de forme analogue au fondamental voisin. (1i) Mesures de masse de noyaux exotiques légers par réaction entre I.L.(7). Les progrès dans la détection et l'identification des ions ont permis des déterminations de masse et d'énergie d'excitatıon dans la région éloignée de la vallée de stabilité, en mesurant avec précision le bilan d'énergie d'une réaction à deux corps de section efficace très faible. Citons les résultats pour ${ }^{1}{ }^{9} \mathrm{~N},{ }^{21} \mathrm{O},{ }^{17} \mathrm{C}$ avec respectivement les réactions $180\left(18_{0}, 19_{\mathrm{N}}\right)^{17} \mathrm{~F}, 18_{0}\left(18_{0}, 15_{0}\right) 21_{0},{ }^{14} \mathrm{C}\left(18_{0},{ }^{17} \mathrm{C}\right)^{15} \mathrm{O}$; aucune de ces mesures ne pouvait être obtenue en i.l. (iii) Etats de couplage coeur-particule du ${ }_{47} \mathrm{Ca}$, peuplés dans la réaction $\left({ }^{3} \mathrm{He}, \alpha\right)$ sur $48 \mathrm{Ca}$ (8). Une série de niveaux de configuration relativement pure correspondant au couplage $2^{+} \otimes 1 \mathrm{f} 7 / 2$ ou $3^{-} \otimes 1 f 7 / 2$ ont été identifiés dans ${ }^{47} \mathrm{Ca}$. Ces états sont atteints par un mécanisme en deux étapes. Les distributions angulaires, caractéristiques du spin sont bien reproduits par un calcul en voies couplées (C.C.R.A.). Cela illustre la présence d'un mécanisme complexe dans une réaction i.l. mais qui pour des structures spécifiques conduit cependant à une sélectivité forte et utilisable. (IV) polarisation du noyau résiduel et mesures de spin (9). Dans des conditions qui sont celles qui justifient le calcul semi-classique, on s'attend à observer une forte polarisation du noyau résiduel $B$, perpendiculairement au plan de réaction. Cela permet de déterminer le spin de $\mathrm{B}^{+}$par une mesure de corrélation entre $\mathrm{b}$ et un produit de désexcitation de $B^{*}$. Une telle corélation, caractéristique du spin de $B$ a été observée dans la réaction ${ }^{16} \mathrm{O}\left(16_{\mathrm{O}},{ }^{12} \mathrm{C}\right){ }^{20} \mathrm{Ne} \rightarrow \alpha+{ }_{\mathrm{O}} \rightarrow{ }^{16}$ à $68 \mathrm{MeV}(9)$. Une mesure comparable bien que fondé sur une polarisation différente $(m=0$ sur l'axe de recul) avait dêjà été observée avec 
${ }^{16} \mathrm{O}\left({ }^{7} \mathrm{~L}_{1}, t\right){ }^{20} \mathrm{Ne} \rightarrow \alpha+16 \mathrm{O}(10)$. Il est intéressant de noter que dans ces deux cas, c'est le mécanisme qui conduit à une polarisation simple alors que dans 1 a méthode de Litherland et Ferguson on n'arrive à une polarisatzon simple ( $m=0$ sur l'axe du faisceau) qu' en s'affranchissant du mécanisme au prix d'une complication expérimentale : la détection à $0^{\circ}$.

(v) Recherche des états de trou de neutron dans les couches profondes par la réaction $\left({ }^{3} \mathrm{He}, \alpha\right)$ (11). Des progrès expérimentaux ont permis d'effectuer un pick-up de neutron par $\left({ }^{3} \mathrm{He}, \alpha\right)$ d'une part à haute énergie ( $607 \mathrm{MeV}$ ) et bonne résolution et d'autre part à moyenne énergie ( $39 \mathrm{MeV}$ ) et très bonne résolution. Il est ainsi devenu possible d'explorer la régzon des couches internes plus profondes et d'étudier la structure fine (fragmentation, étalement) d'états déjà localisés. Du fait de la dynamique de la réaction $\left({ }^{3} \mathrm{He}, \alpha\right)$ qui favorise les transferts élevés $1=3,4,5$ (voir fig. 3 ), cette étude est spécifique des hauts spins. Cette étude est précieuse pour préciser le comportement en profondeur du modèle en couche. (Vi) Strıpping de deux neutrons par I.L. (12). La plupart des études i.1. de stripping de 2 neutrons ont été faites en $(t, p)$. Pour des raisons technıques (machines disponibles pour le tritium) beaucoup ont été faits à basse énergie

(Van de Graff ou "petits" tandems). Le plus souvent, les spectres obtenus s'arrêtent vers quelques $\mathrm{MeV}$ d'énergie d'excitation et pour des ralsons cinématiques et de structure (pairing) les états $0^{+}$sont favorisés. L'utilisation des réactions $(180,160)$ et $\left(14 \mathrm{C},{ }^{12} \mathrm{C}\right)$ a modifié cette situation permettant d'atteinare 10 ou $1.5 \mathrm{MeV}$ d'excitation avec une sélectıvité différente de $(t, p)$, en condussant notamment à des états de particules comportant les deux neutrons dans des couches différentes.

$\mathrm{V}$ - Conclusion.

En dehors des domaines spécifiques respectifs des 1.1. (haute résolution) et des I.L. (muititransferts), il faut insister sur la spécificité du transfert par I.L. du fait de la présence d'un moment orbital interne. Il faut insister aussi sur la nécessité d'explorer la spectroscopie I.L. pour des valeurs du paramètre e (énergie par nucléon au-dessus de la barrière) équivalentes à la gamme des expériences i.l. courantes. Pour cela, l'amélioration de la résolution en énergie est nécessaire malgré la présence d'un facteur cinématique $d E / d \theta$ particulièrement important en I.L. Il est intéressant de noter au résultat expérimental récent (13) montrant la possibilité de "préparer" le faisceau sur la cible (on introduit une corrélation position-énergie et position-angle) de telle sorte que la résolution en énergie se rapproche notablement de celle obtenue avec une émittance de faisceau nulle ou un facteur cisémátique nul.

L'examen dés expériences récentes de spectroscopie par réaction de transfert indique que les domaines des projectiles légers et des projectiles lourds sont également riches. Des progrès expérimentaux élargissent sans cesse les possibilités, particulièrement dans le domaine des ions lourds.

Ce travail doit beaucoup à tous les physiciens qui ont communiqué les résultats mentionnés. C'est par commodité que tous les autres résultats, qui auraient dus être mentionnés, ne l'ont pas été.

\section{Références.}

(1) Direct nuclear reaction theories N. Austern wiley-interscience 1970

(2) Nuclear heavy-1on reactions - P.E. Hodgson Oxford University press 1978

(3) Clustering aspects of nuclear structure and nuclear reactions. Winnipeg 1978 - A.I.P. Conference Proceedings $n^{\circ} 47$

(4) R.D. Amado Phys. Rev. C19 , 1473 (1979)

(5) F. Pougheon, P. Roussel, C. Detraz, G. Rotbard, Phys. Rev. C11 , 287 (1975)

(6) M. Vergnes, G. Rotbard Communication privée et M. Vergnes "Survey of even-even nuclez in the Ge region" conference "Structure of Medium-heavy Nuclez" May 1979 Rhodes, Grèce IPNO - PhN - 79-14

(7) F. Naulin et C. Detraz Communication prıvée et C. Detraz et al. Phys. Rev. C15 (1977) 1738

(8) S. Fortier Communication privée et S. Fortier et al. Nucl. Phys. A311 (1978) 324

(9) F. Pougheon et al. "Study of the $16_{0}\left(16_{0}, 120\right){ }^{20} \mathrm{Ne}$ reaction by polarization measurements' Nuc2. Phys. A325 481

(10) A.D. Panagiotou et al. J. Phys. A7 (1974) 1748

(11) E. Gerlic, communication privée et J. Van de Wiele et al. Nucl. Phys. A297 (1978) 61

(12) M. Bernas, F. Pougheon, M. Roy-Stephan communication privée

(13) E. Kashy, P. Roussel A paraître 